American Journal of Infectious Diseases 2 (1): 18-27, 2006

ISSN 1553-6203

(C) 2006 Science Publications

\title{
The Murine Charged Multivesicular Body Protein 2A, CHMP2A Interacts with the 5' and 3' Terminal Regions of Dengue Virus Complementary Minus-strand RNA
}

\author{
Suchita Chaudhry, Sathyamangalam Swaminathan and Navin Khanna \\ International Centre for Genetic Engineering and Biotechnology, New Delhi 110067, India
}

\begin{abstract}
Dengue (DEN) viruses, of which there are four distinct serotypes (DEN-1, -2, -3 and -4), belong to the Flaviviridae family. Their 'plus' sense RNA genomes contain Non-Translated Regions (NTRs) at their 5' and 3' ends. Replication of viral genomic RNA, which takes place in close association with host cell membranes, involves the initial synthesis of the complementary minus sense RNA intermediate. The NTRs, which have the potential to form stable stem-loop structures, are implicated to play a key role in the viral life cycle through binding to viral and/or host proteins. We have screened a mouse macrophage cDNA expression library with a mixture of radioactive plus and minus sense DEN-2 virus NTR transcripts and identified a $\sim 25 \mathrm{kDa}$ protein. A BLAST analysis of the cDNA sequence encoding this protein showed it to be identical to human CHarged Multivesicular body Protein 2A, CHMP2A (also known as CHromatin Modifying Protein 2A), implicated in sorting proteins into endosome-derived vesicles. Recombinant murine CHMP2A was expressed in E. coli as a 6x His tagged protein, purified to homogeneity and shown to interact preferentially with the minus sense 5' and 3' NTRs of all four DEN virus serotypes. Using DEN-2 virus 5' (-) and 3' (-) NTRs we could demonstrate the specificity of this binding activity in vitro by ultraviolet cross-linking and electrophoretic mobility shift assays, carried out in the absence and presence of specific and nonspecific competitors. Finally, this unique RNA/protein interaction was verified in vivo using a yeastbased interaction assay.
\end{abstract}

Key words: Dengue virus, non-translated regions, chromatin modifying protein 2A (CHMP2A), charged multivesicular body protein, RNA replication

\section{INTRODUCTION}

Dengue (DEN) viruses are mosquito-borne human pathogens with a worldwide prevalence $e^{[1,2]}$. They belong to the family Flaviviridae that also includes Japanese encephalitis, yellow fever, West Nile, Kunjin and hepatitis $\mathrm{C}$ viruses ${ }^{[3]}$. There are four different serotypes of DEN viruses (DEN-1, -2, -3 \& -4), all of which share a common genomic organization. The genome of the DEN virus is a $\sim 11$ kilobase $(\mathrm{kb})$, singlestranded, plus sense RNA that serves as a template for both replication and translation. It contains a single open reading frame (ORF), flanked at its 5' and 3' ends by non-translated regions ( 5 ' and 3 ' NTRs). The single ORF encodes a large, $\sim 3,400$ amino acid (aa) residue polyprotein that is processed during and after translation into three structural proteins called capsid, pre-membrane and envelope and seven nonstructural (NS) proteins, designated as NS1, NS2A, NS2B, NS3, NS4A, NS4B and NS5 $5^{[3]}$. Replication proceeds through a complementary minus sense genomic RNA intermediate. It is well documented that the RNA synthetic machinery of the flaviviruses, like most other eukaryotic cytoplasmic plus-sense RNA viruses, is associated with host cell membrane components ${ }^{[4-6]}$. Mackenzie et al. ${ }^{[7,8]}$ observed for the first time, unique cytoplasmic entities known as vesicle packets (VPs), in DEN-2 virus-infected cells. Subsequently, it was shown that NS1, NS3, NS2A and NS4A (all components of the viral RNA replicase), as well as dsRNA (the putative template for viral RNA synthesis) co-localized to the VPs in Kunjin virus-infected Vero cells ${ }^{[8-10]}$.

The NTRs play key roles in the life cycle of the RNA viruses. The size and sequence of the NTRs vary among different flaviviruses, but their secondary structure comprising stable stem-loop (SL) formations appears to be highly conserved ${ }^{[3,11-13]}$. Translation and replication in the flaviviruses requires interaction between cis-acting elements of the NTRs with viral as well as host proteins ${ }^{[14]}$. While the 5' NTR of the plus sense genomic RNA [5'(+) NTR] is thought to be involved in the initiation of translation, 3' NTR of the plus sense genomic RNA [3'(+) NTR] is implicated to function as a promoter for the synthesis of the complementary minus sense RNA, resulting in a double stranded (ds) replicative form (RF). For example, the cellular proteins, La and polypyrimidine tract binding protein (PTB) have been shown to bind to the $5^{\prime}(+)$ NTR and facilitate translational initiation in hepatitis C virus ${ }^{[15,16]}$. The viral proteins NS3 and NS5, which function as components of the RNA replicase of flaviviruses, have been found to be associated with the

Corresponding Author: Navin Khanna, RGP Laboratory, International Centre for Genetic Engineering \& Biotechnology, PO Box 10504, Aruna Asaf Ali Marg, New Delhi 110067, India 
$3^{\prime}(+) \mathrm{NTR}^{[17]}$. The PTB also binds to the $3^{\prime}(+)$ NTR of HCV RNA ${ }^{[18,19]}$ and may thus affect transcription as well. In the case of West Nile virus, it has been reported that the translation factor EF- $1 \alpha$ binds to the $3^{\prime}(+)$ $\mathrm{NTR}^{[20]}$. Similarly, Japanese encephalitis virus $3^{\prime}(+)$ NTR has been reported to interact with a cellular protein known as Mov34 $4^{[21]}$. Host RNA binding proteins TIAR and TIA-1 involved in translation and splicing are known to interact with 3'(-) NTR of West Nile virus and facilitate plus sense RNA synthesis ${ }^{[22]}$.

To identify DEN virus NTR-interacting proteins we screened a mouse macrophage cDNA expression library with a mixture of radiolabeled DEN-2 $5^{\prime}(+), 3$ ' $(+), 5^{\prime}(-)$ and 3' (-) NTR transcripts and identified a $\sim 25$ kilodalton $(\mathrm{kDa})$ protein that bound specifically to 5'(-) and 3'(-) NTRs of DEN-2 virus but not to their 'plus' sense complementary sequences. The BLAST analysis of the DNA encoding this minus sense NTRbinding protein showed it to be $100 \%$ identical to a murine protein called CHMP-2A, also known as putative breast adenocarcinoma marker protein, BC-2 and vacuolar protein sorting associated protein, $\mathrm{mVps} 2$. The CHMP2A protein is a mammalian class $\mathrm{E}$ Vps (vacuolar protein sorting) protein involved in the endosome related multivesicular body (MVB) sorting pathway ${ }^{[23,24]}$. Interestingly, all characterized positivestrand RNA viruses are believed to assemble their RNA replication complexes on intracellular membranes, usually in association with membrane vesicle formation ${ }^{[6]}$. This is the first report to describe the identification of a host protein that interacts with the 5 , and 3' NTRs of DEN-2 complementary minus strand RNA.

\section{MATERIALS AND METHODS}

DNA templates and RNA transcripts: The cDNAs corresponding to DEN-2 virus (Genbank accession No. AY744150) 5'(+) NTR [nucleotides (nt) 1-96] and $3^{\prime}(+) \quad$ NTR (nt 10270-10714) were chemically synthesized (Biobasic Inc. Toronto, Canada), so that the cDNA fragments were flanked by $\mathrm{Kpn}$ I and Sac I sites at their 5' and 3' ends, respectively. The 5' NTR [96 base pairs (bp)] and 3' NTR (444 bp) encoding cDNA inserts were cloned into plasmid pBluescript $\mathrm{KS}+$ at these sites, to create plasmids pBSD2-5 and pBSD2-3, respectively. These NTR cDNA inserts were amplified together with the vector provided $\mathrm{T} 3$ and $\mathrm{T} 7$ phage promoter sequences using polymerase chain reaction (PCR) to obtain templates (5' NTR templates: $135 \mathrm{bp}$; 3' NTR templates: $483 \mathrm{bp}$ ) for in vitro transcription. The vector-specific forward primers [designed to anneal to the T7 or T3 RNA polymerase promoter sites) and the NTR insert-specific reverse primers and their sequences, are as follows: primer A, aat taa ccc tca cta aag gga ac; primer $\mathrm{B}$, cag aga tct gct ctc taa tca aaa; primer $\mathrm{C}$, gta ata cga ctc act ata ggg $c$; primer $\mathrm{D}$, agt tgt tag tct acg tgg acc g; primer E, aga acc tgt tga ttc aac agc ac; and primer F, tag aaa aac atg aga cag aac aa a ag. Primers were obtained from Operon, Germany. The templates and primer pair combinations used for PCR are shown in Fig. 1. The PCR products were directly used as templates to synthesize radiolabeled RNA by in vitro transcription with either $\mathrm{T} 7$ or $\mathrm{T} 3$ RNA polymerase (Roche Applied Science). To generate $\left[{ }^{32} \mathrm{P}\right]$-labeled RNA, the transcription reactions were carried out in presence of $40 \mathrm{mM}$ Tris- $\mathrm{Cl}(\mathrm{pH}$ 7.5), $6 \mathrm{mM} \mathrm{MgCl} 2,10 \mathrm{mM} \mathrm{NaCl}, 2 \mathrm{mM}$ spermidine, 10 $\mathrm{mM}$ dithiothreitol (DTT), $0.5 \mathrm{mM}$ (each) ribonucleotides (A, C and G), 10 $\square \mathrm{M}$ UTP, $50 \square \mathrm{Ci}$ of $\alpha\left[{ }^{32} \mathrm{P}\right]-\mathrm{UTP}(3000 \mathrm{Ci} / \mathrm{mmol}$; Perkin Elmer) and $20 \mathrm{U}$ of RNasin (Promega). The reaction mixture was incubated for $2 \mathrm{~h}$ at $30^{\circ} \mathrm{C}$. RNase-free DNase $1(0.2 \mathrm{U})$ was then added and reaction mixture was incubated at $37^{\circ} \mathrm{C}$ for $30 \mathrm{~min}$. This reaction was followed by one phenolchloroform-isoamyl alcohol and one chloroformisoamyl alcohol extraction. To remove free nucleotides, the aqueous phase containing the RNA was passed through a Sephadex G-25 spun column and stored at $70^{\circ} \mathrm{C}$. For generating radiolabeled and photo-reactive transcripts to be used in ultraviolet cross-linking (UV$\mathrm{CL})$ experiments, 5-Bromo-Uridine Triphosphate $(\mathrm{Br}-$ UTP) was added (instead of UTP) to the transcription reaction. RNA yield was determined by trichloroacetic acid precipitation. A specific activity of $\sim 10^{8} \mathrm{cpm} / \mu \mathrm{g}$ was routinely obtained. For synthesis of cold RNA, the reaction was carried out as essentially described above except that $0.5 \mathrm{mM}$ cold UTP replaced the radiolabeled UTP. All the reagents used were made in diethyl pyrocarbonate (DEPC) treated water.

Cloning of a DEN virus NTR-binding protein from a mouse macrophage cDNA library: A commercially available cDNA expression library (Stratagene), made from mouse macrophages in the Uni-ZAP XR vector, was screened using a mixture of radiolabeled DEN virus NTR transcripts, according to the manufacturer's protocol. The membranes were incubated at room temperature for $30 \mathrm{~min}$ in binding buffer $[14 \mathrm{mM}$ HEPES ( $\mathrm{pH}$ 7.5)/6 mM Tris-CI ( $\mathrm{pH}$ 7.5)/1 mM EDTA/1 mM DTT/60 mM KCI and $10 \mu \mathrm{g} \mathrm{ml}^{-1}$ each of yeast tRNA, yeast RNA, synthetic poly A, poly AU, poly IC and $100 \mu \mathrm{g} \mathrm{ml}^{-1}$ of sheared salmon sperm DNA]. The membranes were probed with an equimolar mixture of $\alpha-\left[{ }^{32} \mathrm{P}\right]$-radiolabeled DEN-2 virus $5^{\prime}(+), 3^{\prime}$ $(+), 5^{\prime}(-)$ and $3^{\prime}(-)$ NTR transcripts $\left(\sim 1 \times 10^{6} \mathrm{cpm}\right.$ of each probe/ml). The putative positive plaques were visualized by autoradiography, picked up and purified to homogeneity ${ }^{[25]}$. In vivo excision and recircularization of cloned inserts contained within the lambda vector into corresponding pBluescript doublestranded phagemids was carried out as per the manufacturer's protocol. The phagemids were then sequenced, using $\mathrm{T} 3$ and $\mathrm{T} 7$ primers (flanking the cDNA inserts).

Recombinant expression of the DEN virus NTRbinding protein in $E$. coli: $\mathrm{T} 3-$ and $\mathrm{T} 7$-primer 
amplified insert from a phagemid clone selected above was T/A cloned into pGEM-T Easy vector, retrieved as an EcoR I fragment and inserted into pBluescript at its $E c o R$ I site. After identifying a clone containing the insert in the right orientation, it was recovered as a Sma I and Hind III fragment and cloned directionally into the $E$. coli expression vector pQE31 (Qiagen), in-frame with the vector-provided $6 \mathrm{x}$ His tag-encoding sequence at the 5' side. The insert carried its own stop codon. The resultant pQE-derived expression plasmid encoding the NTR-binding protein was introduced into E. coli (SG13009) cells and induced with $0.5 \mathrm{mM}$ IPTG for $4 \mathrm{hrs}$ at $37^{\circ} \mathrm{C}$. The protein was purified by Ni-NTA chromatography as described earlier ${ }^{[26]}$. The bound protein was eluted at $\mathrm{pH} 4.5[10 \mathrm{mM}$ Tris- $\mathrm{HCl} / 100$ $\mathrm{mM} \mathrm{NaH} \mathrm{PO}_{4} / 8 \mathrm{M}$ urea, $\mathrm{pH} 4.5$ ) and dialyzed against $10 \mathrm{mM}$ Tris, pH7.5/50 mM L-arginine/50 mM Lglutamic acid.

Binding of Ni-NTA immobilized r-mCHMP2A to DEN virus NTR transcripts: Purified r-mCHMP2A protein $(\sim 100 \mathrm{ng})$ was allowed to bind Ni-NTA agarose beads ( $50 \mu \mathrm{l}$ of a $10 \%$ suspension), pre-equilibrated in binding buffer [5 mM HEPES (pH 7.5), 1 mM EDTA, $2 \mathrm{mM}$ DTT, $25 \mathrm{mM} \mathrm{KCl}, 2 \mathrm{mM} \mathrm{MgCl}_{2}, 4 \%$ (vol/vol) glycerol]. The beads were spun down, washed in binding buffer and allowed to incubate with $\sim 1 \mathrm{ng}$ $\left(10^{5} \mathrm{cpm}\right)$ of radiolabeled NTR or pBS polylinker transcript, in the presence of $200 \mathrm{ng}$ each of yeast tRNA, synthetic poly A, poly AU and poly IC, for $1 \mathrm{hr}$ on ice. The beads were then washed extensively binding buffer to remove unbound radioactivity and analyzed using a liquid scintillation counter to determine the magnitude of bound radioactivity.

Ultraviolet light-induced cross-linking (UV-CL) of DEN-2 NTRs and the purified recombinant protein: One hundred nanograms of the purified r-mCHMP2A, unrelated 6x Histidine tagged protein (r-human IFNgamma), or bovine serum albumin were incubated in UV-CL binding buffer [5 mM HEPES ( $\mathrm{pH} 7.5$ ), $1 \mathrm{mM}$ EDTA, $2 \mathrm{mM}$ DTT, $25 \mathrm{mM} \mathrm{KCl,} 2 \mathrm{mM} \mathrm{MgCl}_{2}, 4 \%$ (vol/vol) glycerol] with $200 \mathrm{ng}$ each of yeast tRNA, synthetic poly A, poly AU and poly IC (Sigma) in the presence of $1 \mathrm{U}$ RNasin (Roche Applied Sciences) in a final volume of $20 \mu \mathrm{l}$ for $10 \mathrm{~min}$ at $4^{\circ} \mathrm{C}$. To this, $1 \mathrm{ng}$ of $\square-\square{ }^{32} \mathrm{P}$ ]-labeled DEN-2 NTR transcript [either 5'(+/-) NTR or 3'(+/-) NTR] was added and incubated for another $15 \mathrm{~min}$ at $4^{\circ} \mathrm{C}$. The reaction mixture was then irradiated at a distance of $5 \mathrm{~cm}$, with $254 \mathrm{~nm}$ UV light (Stratalinker) for $2 \mathrm{~min}$ on ice. Samples were treated with $2.5 \mathrm{U}$ of RNase $\mathrm{A} / \mathrm{T} 1$, for $30 \mathrm{~min}$ at $37^{\circ} \mathrm{C}$ and subjected to $12.5 \%$ SDS-PAGE. After electrophoresis, gels were fixed, dried and autoradiographed to visualize the UV cross-linked protein bands. To evaluate the specificity of RNA-protein interactions, UV induced cross-linking was also carried out with 20 -fold molar excess of unlabeled homologous and unrelated competitor transcripts.

In vivo interaction between purified recombinant protein and DEN 2 virus 5'(-) NTR through yeast three-hybrid assay: Competent yeast cells (strain L-40 ura3) were co-transformed with the RNA bait hybrid plasmid (plasmid pRH5' with DEN-2 virus 5' (+), 3' (+), 5' (-), 3' (-) NTRs or pBS polylinker cloned at Pme I and Aat II sites), prey plasmid (plasmid pYESTrp3 with the murine macrophage gene encoding the 222 aa ORF, cloned at EcoR I site) and plasmid pHybLex/Zeo-MS2 (RNA-Protein Hybrid hunter kit, Invitrogen life technologies) and selected on plates lacking uracil, tryptophan and histidine in the presence of $100 \mathrm{mM}$ zeocin and $5 \mathrm{mM} \mathrm{3-AT}$. The resultant positive colonies were streaked on appropriate selection plates and transferred to Whatman filter discs. After marking the orientations the transferred yeast colonies were permeabilized by freeze thaw cycles using liquid nitrogen. The filter discs were then carefully placed with colony side up on the filters presoaked in $\mathrm{Z}$ buffer (60 mM Na $\mathrm{HPO}_{4} \cdot 7 \mathrm{H}_{2} \mathrm{O} / 140 \mathrm{mM} \quad \mathrm{NaH}_{2} \mathrm{PO}_{4} \cdot \mathrm{H}_{2} \mathrm{O} / 10$ $\mathrm{mM} \mathrm{KCl} / 10 \mathrm{mM} \mathrm{MgSO} 4.7 \mathrm{H}_{2} \mathrm{O}$ ) with $0.27 \mathrm{ml} \beta$ mercaptoethanol and $1.67 \mathrm{ml}$ of 5-bromo-4-chloro-3indolyl- $\beta$-D-galactopyranoside (x-gal: $20 \mathrm{mg} / \mathrm{ml}$ ) per $100 \mathrm{ml}$ of $\mathrm{Z}$ buffer and incubated at $30^{\circ} \mathrm{C}$ for $7-8 \mathrm{hrs}$, till blue colonies appeared.

Preparation of whole cell lysate and electrophoretic mobility shift assay (EMSA): BHK-21 cells), were maintained in Dulbecco's modified Eagle medium containing $10 \%$ fetal calf serum at $37^{\circ} \mathrm{C}$ in a humidified $\mathrm{CO} 2$ incubator. TWhole cell lysates of BHK cells were prepared as follows. BHK monolayer in a T-75 flask $\left(\sim 5 \times 10^{7}\right.$ cells $)$ was washed twice with phosphate buffer saline (PBS) and harvested in $\sim 1 \mathrm{ml} \mathrm{PBS}$, using a cell scraper. The cells were then centrifuged at $1,000 \mathrm{rpm}$ and lysed by re-suspending in in $500 \mu \mathrm{l}$ of lysis buffer (10 mM HEPES pH7.9, 10 mM NaCl, 1\% Triton $\mathrm{x}-100$, $1 \mathrm{mM}$ DTT, 5\% glycerol and protease inhibitors) followed by 5 freeze-thaw cycles of 1 min each. The resulting lysate was clarified by centrifugation at $13,000 \mathrm{rpm}$ for $1 \mathrm{hr}$ at $4^{\circ} \mathrm{C}$. The supernatant (whole cell lysate) was carefully separated and its protein concentration determined by BCA method using bovine serum albumin as a standard. For EMSA, $\sim 10 \mu \mathrm{g}$ whole cell lyasate protein from uninfected BHK cells was incubated in binding buffer [5 mM HEPES ( $\mathrm{pH} 7.5) / 1$ $\mathrm{mM}$ EDTA/2 mM DTT/25 mM KCI/2 mM MgCl$/ 2 \%$ glycerol (v/v)/50U RNasin/ml] with $10^{5} \mathrm{cpm}$ of $\alpha-\left[{ }^{32} \mathrm{P}\right]-$ labeled RNA probe ( $\sim 1$ ng NTR transcripts), in the presence of $100 \mathrm{ng}$ yeast tRNA, in a final volume of 20 $\mu \mathrm{l}$, for $15 \mathrm{~min}$ at $4^{\circ} \mathrm{C}$. RNA-protein complexes were electrophoresed at $4^{\circ} \mathrm{C}$ on a non-denaturing $6 \%$ polyacrylamide gel (acrylamide/bisacrylamide ratio, 40:1) in $0.25 \mathrm{x}$ Tris-borate-EDTA buffer at constant amperage of $10 \mathrm{~mA}$. The gel was then dried and the complexes were visualized by autoradiography. 


\section{RESULTS}

The identification of murine charged multivesicular body protein $2 \mathrm{~A}$ (or chromatin modifying protein 2A) as a DEN-2 NTR-binding protein: Two plasmids, both based on pBluescript $\mathrm{KS}+$, were constructed by cloning in chemically synthesized cDNAs, corresponding to either the 5' or 3' NTR of DEN-2 virus, between the vector provided phage T3 and T7 promoters, into Kpn I and Sac I sites. These sites were chosen so that transcripts generated will have the minimum number of vector-encoded nucleotides. The resultant T3-NTR-T7 cassettes are shown schematically in Fig. 1A (top). The NTR-encoding cDNAs from these two plasmids were amplified to generate two different PCR products each, one, which included the vector provided T7 promoter sequences and the other, which included the T3 promoter sequences. These four PCR products (I to IV, Fig. 1A) were used as templates for in vitro transcription, in the presence of $\alpha-\left[{ }^{32} \mathrm{P}\right]-\mathrm{UTP}$, to generate radiolabeled RNA transcripts corresponding to DEN-2 virus 5' and 3' NTRs, of both plus and minus sense. T3 RNA polymerase-mediated in vitro transcription, in the presence of $\alpha-\left[{ }^{32} \mathrm{P}\right]-\mathrm{ATP}$, generated a 461 base3' $^{\prime}+$ ) NTR transcript (Fig. 1B, lane 1) and a 113 base5'(+) NTR transcript (Fig. 1B, lane 2). Similarly, T7 RNA polymerase transcription resulted in the generation of 5'(-) and 3'(-) DEN-2 NTRs (Fig. 1B, lanes 3 and 4 respectively).

We next used a mixture of all four radiolabled DEN-2 NTR transcripts to screen a mouse macrophage cDNA expression library. DEN viruses are known to replicate in human macrophages and mouse macrophages cDNA expression library is commercially available. About one million recombinant plaques from the mouse macrophage library were screened to identify recombinant bacteriophages that repeatedly bound to the DEN-2 NTR mix. A total of 16 bacteriophage clones, obtained after quaternary screening, were plaque purified four times before being used for rescuing the cDNA inserts in a plasmid form. Upon PCR using T3 and T7 primers, amplification products of sizes, $\sim 0.6$ ( 1 clone), $\sim 0.8$ (1 clone), 1.2 (4 clones) and 1.5 (10 clones) $\mathrm{Kb}$ were obtained. Four representative clones corresponding to each of the observed sizes were sequenced in both directions. The sequences obtained were subjected to BLAST search and results are tabulated in Table 1 . All clones, but one, represented partial sequences (Cap-Z $\beta$ and RuvBL-2) and were not pursued further in this study. Efforts are in progress to obtain their complete sequence and to further study their interaction with DEN NTRs. However, one single clone, the $\sim 0.6 \mathrm{~Kb}$ cDNA, contained a complete ORF encoding a 222 amino acid protein with a predicted molecular mass of $\sim 25 \mathrm{kDa}$. BLAST search revealed that this DEN virus NTRbinding protein is $100 \%$ identical to murine CHarged Multivesicular body Protein 2A (or CHromatin
Modifying Protein 2A), mCHMP2A $\mathrm{A}^{[23,24]}$, also known as the breast carcinoma marker protein, $\mathrm{BC}-2^{[27]}$ and $\mathrm{mVps} 2 \mathrm{p}^{[24]}$. This protein shares $\sim 99.5 \%$ identity with a human homologue hCHMP2A, with just a single aa residue difference in the $\mathrm{C}$-terminal region and $\sim 45 \%$ identity with the yeast homologue, Did4p (also known as Chm2p), as depicted in the Clustal W alignment (Fig. 2). Interestingly, all these proteins belong to class E Vps (vacuolar protein sorting) proteins, which are involved in sorting proteins into vesicles that bud into multivesicular bodies (MVB) ${ }^{[28]}$.

Expression and purification of recombinant mCHMP $2 \mathrm{~A}$ protein: The $\sim 0.6 \mathrm{~Kb}$ gene encoding $\mathrm{mCHMP} 2 \mathrm{~A}$ protein $(m C H M P 2 A)$ was inserted in-frame with the translation initiator codon and an aminoterminal $6 x$ histidine tag-encoding sequence of the bacterial expression vector pQE31. This expression vector is depicted in Fig. 3A. Figure 3B shows the induction profile of a typical clone. It is evident that induction of recombinant $m C H M P 2 A(r-m C H M P 2 A)$ gene expression using IPTG was accompanied by the appearance of a new $\sim 28 \mathrm{kDa}$ band, consistent with the predicted size of the r-mCHMP2A protein (Fig. 3B, lane 2).

A lysate prepared from induced cells was chromatographed on a Ni-NTA column under denaturing conditions as shown in Fig. 3C. Under the experimental conditions employed, all of the induced protein bound to the column, as evident from the comparison of polypeptide profile of the initial lysate loaded on the affinity matrix (L) with that of the flowthrough material (FT). After washing the column extensively with pH 6.3 and pH 5.9 buffers (W), elution with a $\mathrm{pH} 4.5$ buffer resulted in the emergence of highly purified ( $>95 \%$ pure), recombinant protein from the column (E). To remove the urea from the eluted protein, the eluted fractions were pooled and dialyzed against $10 \mathrm{mM}$ Tris $\mathrm{pH} 7.5$, containing $50 \mathrm{mM}$ Larginine and $50 \mathrm{mM}$ L-glutamic acid ${ }^{[29]}$. It has been reported that this buffer maintains the charged state of the protein and keeps it in soluble form. However, this resulted in partial precipitation of the protein $(\mathrm{P})$ with $\sim 30 \%$ remaining soluble $(\mathrm{S})$, as shown in Fig. 3C. The protein concentration was $\sim 0.5 \mathrm{mg} / \mathrm{ml}$. We used this soluble fraction for further investigation.

Recombinant $\mathrm{mCHMP2A}$ protein binds to Dengue virus 5' (-) and 3' (-) NTRs, but not to their plus sense complements: As the mCHMP $2 \mathrm{~A}$ protein was initially identified based on its ability to bind to a mixture of four NTRs, we sought to investigate the capacity of the E. coli-expressed recombinant protein to bind to each of these four NTRs separately. His-tagged r-CHMP2A protein ( 100 ng) was immobilized onto Ni-NTA beads and incubated, separately, with $1 \mathrm{ng}$ $\left(\sim 10^{5} \mathrm{cpm}\right)$ each of the four radiolabeled DEN-2 NTR transcripts in UV cross-linking buffer in the presence 
Table 1: BLAST analysis of sequences obtained from mouse macrophage cDNA expression library screened using a mixture of four probes corresponding to DEN NTRs

\begin{tabular}{|c|c|c|c|c|}
\hline \multirow[t]{2}{*}{ Gene and Proposed Functions } & \multirow{2}{*}{$\begin{array}{l}\text { ORF present in cDNA } \\
\text { insert }\end{array}$} & \multirow{2}{*}{$\begin{array}{l}\text { Protein } \\
\text { mass } \\
(\mathrm{kDa})\end{array}$} & \multicolumn{2}{|c|}{ Homology } \\
\hline & & & Mouse & Human \\
\hline $\begin{array}{l}\text { Charged Multivesicular Body Protein 2A (CHMP2A) Component of } \\
\text { ESCRT complex involved in transport and sorting of endosomal cargo } \\
\text { protein, chromatin condensation during cell cycle }\end{array}$ & $\begin{array}{l}\text { Complete ORF } \\
\text { (222 aa) }\end{array}$ & 25 & 99 & 99 \\
\hline $\begin{array}{l}\text { Actin filament capping protein }(\mathrm{Cap}-\mathrm{Z} \beta) \\
\text { Actin capping protein that regulates growth of actin filaments by capping } \\
\text { barbed ends of growing actin filaments }\end{array}$ & $\begin{array}{l}\text { Partial ORF } \\
\text { (C-terminal } 11 \text { aa) }\end{array}$ & 32 & 99 & 92 \\
\hline $\begin{array}{l}\text { E.coli UV-repair like protein (Ruv BL-2) } \\
\text { Single-strand DNA stimulated ATPase and ATP-dependent helicase, } \\
\text { involved in transcription and recombination }\end{array}$ & $\begin{array}{l}\text { Partial ORF } \\
\text { (C-terminal } 382 \text { aa) }\end{array}$ & 51 & 99 & 95 \\
\hline
\end{tabular}

Abbreviations: ORF, Open reading frame. aa, amino acids. ESCRT, Endosomal sorting complexes required for transport.

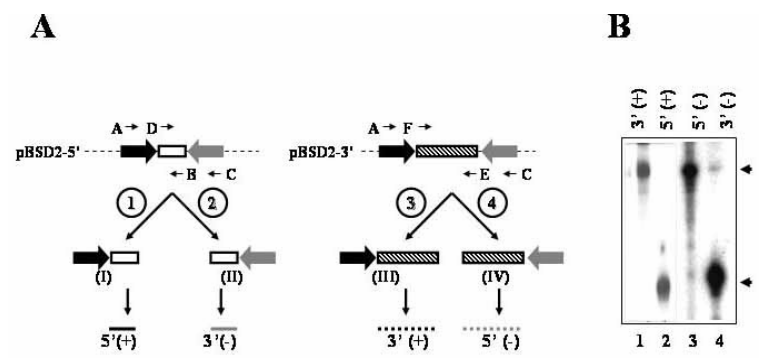

Fig. 1: Strategy for the generation of 'plus' and 'minus' sense DEN virus NTR RNA transcripts. (A) Plasmids, pBSD2-5 and pBSD2-3 were constructed by inserting DEN-2 virus 5' NTR (indicated by the open box)- and 3' NTR (indicated by the hatched box)-encoding cDNAs between phage T7 (left pointing grey arrow) and T3 (right pointing black arrow) promoters of pBluescript. These plasmids were used as templates in four PCR reactions (numbered 1-4). The tiny arrows above and below the T3-NTR-T7 cassette represent primers (indicated as A-F). Primers A and $\mathrm{C}$ are T3 and T7 promoter primers, respectively. Primers D and B are 5'NTR cDNA-specific forward and reverse primers, respectively. Similarly, primers F and E are 3'NTR cDNA-specific forward and reverse primers, respectively Amplification of plasmid pBSD2-5, with primer pairs $\mathrm{A}+\mathrm{B}$ (reaction 1) and $\mathrm{C}+\mathrm{D}$ (reaction 2) produced PCR products I and II, respectively. Similarly, amplification of pBSD2-3 with primer pairs A+E (reaction 3 ) and $\mathrm{C}+\mathrm{F}$ (reaction 4 ) generated PCR products III and IV, respectively. In vitro transcription of these PCR products (I and III with T3 RNA polymerase; II and IV with T7 RNA polymerase) resulted in the generation of the NTR transcripts (short solid lines denote 5' NTRs; the longer dashed lines indicate the 3' NTRs; the plus sense NTRs are shown in black and the minus sense NTRs in grey). (B) Autoradiogram of an $\alpha-\left[{ }^{32} \mathrm{P}\right]$-labeled in vitro transcripts; 461 base $3^{\prime}(+)$ NTR (lane 1) and 113 base5' (+) NTR (lane 2) and the complimentary $5^{\prime}(-)$ NTR (lane 3 ) and 3'(-) NTR (lane 4) generated using the PCR products III, I, IV and II, respectively, in panel A as the templates. DNA size markers were run in lane 1 ( $1 \mathrm{~kb}$ ladder) and lane 4 (123 bp ladder) of panels A and $\mathrm{B}$. The arrows to the right of the panel denotes in vitro synthesized NTR transcripts

RNasin (1U) and non-specific competitors (200 ng each of yeast tRNA, synthetic poly A, poly AU and poly IC). For comparison, we also analyzed the interactions of NTRs corresponding to the remaining three DEN serotypes in the same experiment. After extensive washing, the bound radioactivity was measured. To correct for non-specific binding, we used $1 \mathrm{ng}$ of

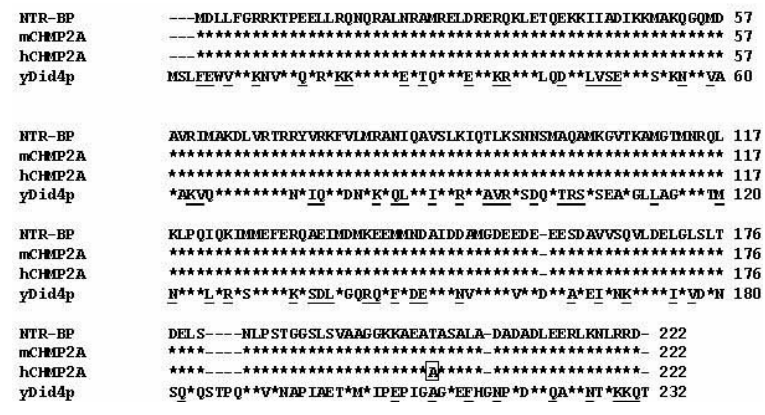

Fig. 2: ClustalW sequence alignment of the macrophage DEN-2 virus 5'(-) NTR-binding protein with other eukaryotic class E Vps proteinsmouse CHMP2A/BC-2/Vps2p, human CHMP2A and yeast $\mathrm{Did} 4 / \mathrm{Chm} 2$ proteins. The amino acid sequence of the NTR-binding protein (NTR-BP) identified in this study was predicted from the nucleotide sequence of the cDNA. Sequences for murine CHMP2A (mCHMP2A, Acc. No. AK005267), human CHMP2A (hCHMP2A, Acc. No. BC002502) and yeast Did4 (yDid4, Acc. No. P36108) were retrieved from the GenBank (accession numbers are shown in parantheses). ClustalW multiple sequence alignment was performed using the online tool available at the European Bioinformatics Institute website (http://www.ebi.ac.uk/). Stars indicate complete identity. Conserved residues are underlined. The boxed residue in the hCHMP2A sequence indicates the single difference between the murine and human proteins. Dashes indicate gaps introduced to maximize alignment

radiolabeled transcript generated from the polylinker of the pBluescript plasmid without any cloned NTRs. The results depicted in Fig. 4 demonstrated that, rmCHMP2A (in the presence excess of non-specific RNA), bound to both 5' (-) and 3' (-) NTRs of all four DEN virus serotypes to a significant degree. Binding to 5' (+) and 3' (+) NTRs in most instances was barely discernible. No binding of labeled NTRs was observed when $\mathrm{r}$-mCHMP2A was replaced with an unrelated Histidine-tagged human IFN- $\gamma$ or bovine serum albumin (data not shown).

We carried out UV cross-linking experiments to investigate this interaction further. To this end, DEN-2 NTR transcripts [5'(-), 3'(-), 5'(+) and $\left.3^{\prime}(+)\right]$ were generated from corresponding templates in the presence of Br-UTP and $\alpha-\left[{ }^{32} \mathrm{P}\right]-\mathrm{ATP}$ to render them both photoreactive and radioactive. One ng of each of these 4 
$\mathbf{A}$

B
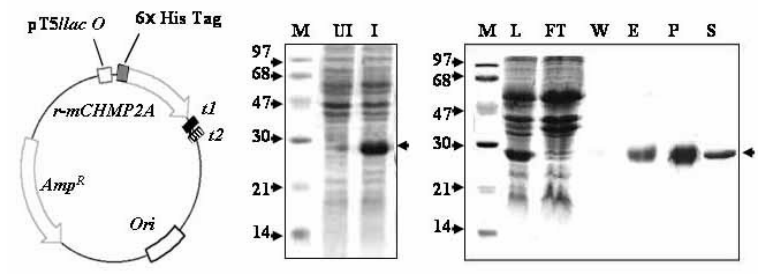

Fig. 3: Expression of recombinant mCHMP2A protein in E. coli. (A) Map of the mCHMP2A expression plasmid. (B) Coomassie stained denaturing gel showing the polypeptide profiles of E. coli harboring the plasmid in A. (C) SDSPAGE analysis of column fractions. Affinity purified $\mathrm{r}$ mCHMP2A was dialyzed (against a neutral buffer containing $50 \mathrm{mM}$ each of L-glutamic acid and L-arginine), to remove the urea. The dialyzed material was centrifuged and equivalent amounts of the pellet and supernatant were analyzed. (M, Molecular weight markers; UI, Un-induced; I, Induced; L, Load; FT, Flow through; W, wash; E, Elution; P, Pellet; S, Soluble)

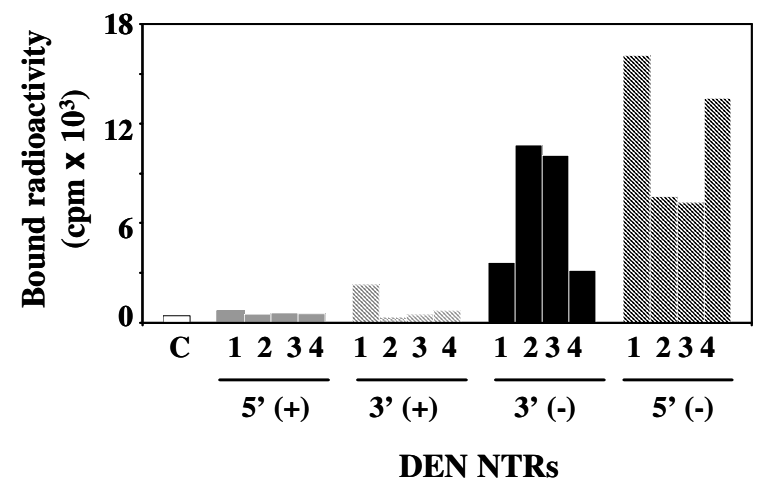

Fig. 4: Binding of purified r-mCHMP2A to the + and - sense NTRs of all four DEN virus serotypes. Purified r-mCHMP2A protein (100 ng) was immobilized on Ni-NTA agarose beads and incubated in the presence of non-specific RNA (200 ng each of yeast tRNA, synthetic poly A, poly AU and poly IC) with $1 \mathrm{ng}$ of $\alpha$-[ $\left.\mathrm{P}^{32}\right]$-labeled, in vitro synthesized RNA transcripts corresponding to $5^{\prime}(+)$ (solid grey bars), $3^{\prime}(+)$ (hatched grey bars), 3'(-) (solid black bars) and 5'(-) (hatched black bars) NTRs, of each of the four DEN virus serotypes (indicated by 1, 2, 3 and 4 in the horizontal axis). A control transcript (1 ng) (empty bar), corresponding to the pBS polylinker was assayed in parallel, to correct for nonspecific background. The histogram shows the bound radioactivity (average of two independent experiments) determined after extensive washing of the beads

transcripts and an unrelated control transcript were then incubated with $\mathrm{r}-\mathrm{mCHMP} 2 \mathrm{~A}$, in presence of excess of non-specific competitors, in solution. Following UVirradiation to promote cross-linking, samples were digested with RNase as described in materials and methods and analyzed by SDS-PAGE. Both 5'(-) and 3'(-) NTR transcripts generated radiolabeled UV-CL complexes on the gel (Fig. 5A). Once again, rmCHMP2A did not manifest any significant binding to either 5'(+) or 3'(+) NTR. These results, obtained once again in the presence of non-specific competitors, essentially mirrored the binding assay data in Fig. 4 and

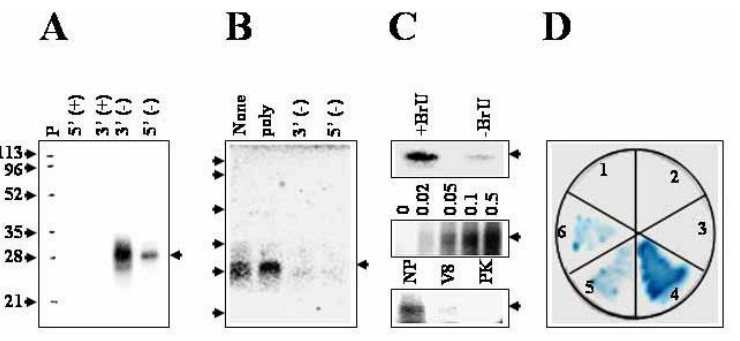

Fig. 5: In vitro and in vivo analyses of the binding of mCHMP2A to DEN-2 virus plus and minus sense NTRs. (A) This panel depicts a UV-CL experiment wherein $100 \mathrm{ng}$ of pure $\mathrm{r}-$ mCHMP2A protein was incubated, separately, with $1 \mathrm{ng}$ each of $5^{\prime}(+), 3^{\prime}(+), 3^{\prime}(-)$ and 5' (-) radiolabeled DEN-2 virus NTRs, in the presence of excess of non-specific competitors. Samples were UV-irradiated for $2 \mathrm{~min}$, treated, with RNase A and RNase T1 for 30 min run on SDS-PAGE and autoradiographed. The positions of pre-stained protein size markers (lane $\mathrm{p}$ ) were traced with radioactive ink prior to autoradiography. Their sizes (in $\mathrm{kDa}$ ) are indicated to the left. (B) UV-CL experiment performed in the presence of competitors. A routine UV-CL experiment (all details as in A) performed using r-mCHMP2A and DEN-2 virus 3' (-) NTR in the absence of any added competitor (lane 'none'). The same experiment was performed in parallel using $\mathrm{pBS}$ polylinker transcript (lane 'poly'), 3' (-) NTR transcript [lane "3'(-)"] and 5' (-) NTR transcript [ lane "5' (-)"] as competitors. All competitor transcripts were unlabeled and at $\sim 20 \mathrm{x}$ molar excess. (C) DEN-2 virus 5'(-) NTR/r$\mathrm{mCHMP} 2 \mathrm{~A}$ complex is visualized in the UV-CL experiment only if it is carried out with the RNA transcript made in presence of Br-UTP (lane 1, top panel) and not in the absence of Br-UTP (lane 2, top panel); the intensity of UVCL complex increases with the amount of r-mCHMP2A (middle panel; the numbers on top indicate the $\square \mathrm{gs}$ of protein used); and the UV-CL complex (shown with $0.1 \mathrm{ug}$ protein) is sensitive to V8 protease (lane 'V8', bottom panel) and proteinase $\mathrm{K}$ (lane ' $\mathrm{PK}$ ', bottom panel) and is discernible in their absence (no protease, lane 'NP', bottom panel). (D) The plate depicts the results of a typical yeast three-hybrid assay. Yeast transformants harboring $r$ mCHMP2A prey construct together with any one of five bait constructs [pBS polylinker transcript (sector 1); 5' (+) NTR (sector 2); 3' (+) NTR (sector 3); 5' (-) NTR (sector 5); and 3' (-) NTR (sector 6); all NTRs were from DEN-2 virus] were streaked on a selection plate and transferred to a Whatmann disc and processed for $\tilde{\beta}$ galactosidase expression. A yeast transformant harboring positive control constructs [bait plasmid encoding iron response element (IRE) RNA/prey plasmid encoding IRE-binding protein,], provided with the Invitrogen RNA-Protein Hybrid Hunter kit, was analyzed in parallel (sector 4)

suggest that the r-CHMP2A protein binds to both 5'(-) and 3'(-) NTRs specifically and preferentially. To corroborate this conclusion, we carried out competition experiments as shown in Fig. 5B. In this experiment, the UV-CL complex was formed between radiolabeled 3'(-) NTR and r-CHMP2A (lane 'None'). The formation of this complex was unaffected (lane 'poly') by the presence of a twenty-fold molar excess of unlabeled, control RNA transcript (corresponding to the MCS sequence of pBluescript). Interestingly, the formation of this complex was abrogated by the presence of an equivalent amount of either unlabeled 3'(-) NTR [lane “3'(-)"] or unlabeled 5'(-) NTR [lane 
A B C

D

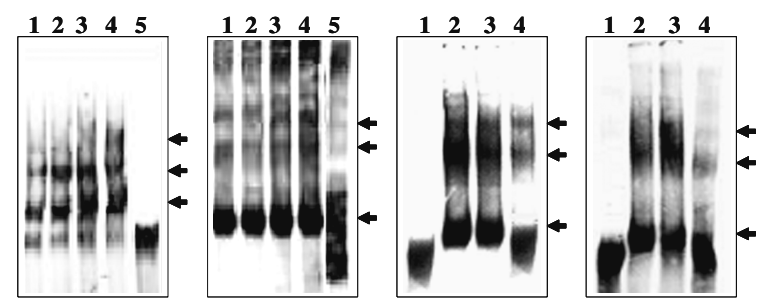

Fig. 6: BHK cells contain proteins that can specifically interact with DEN-2 virus 5' (-) and 3' (-) NTRs. Panels A and B: Electrophoretic mobility shift assays (EMSA) were carried out using BHK cell lysate and either radiolabeled 3' (-) NTR transcripts (A) or 5' (-) NTR transcripts (B). In both panels A and B, NTRs corresponding to all four DEN serotypes (indicated above the lanes in Arabic numerals) were used. Free DEN-2 virus 3' (-) NTR and 5' (-) NTR probes (in the absence of any BHK proteins) were run in lane 5 of panels A and $\mathrm{B}$, respectively. Panels $\mathrm{C}$ and D: EMSAs with BHK lysates and DEN-2 virus 5' (-) NTR (C) or DEN-2 virus 3' (-) NTR (D) carried out in the presence of unlabeled non-specific (lanes 3, panels $\mathrm{C}$ and D) or homologous NTR transcripts (lanes 4, panels $\mathrm{C}$ and D) as competitors (at 20x molar excess). Control experiments in the absence of competitors were run in lanes 2 (panels $C$ and D). Free DEN-2 5' (-) NTR and 3' (-) NTR probes were run in lanes 1 (panels C and D). The arrows to the right of the panels indicate the RNA/protein complexes

5'(-)"]. The data in this experiment reinforce the notion that the interaction between the minus sense NTRs and rCHMP2A is indeed specific. Additionally, it also suggests that both the 5'(-) and 3'(-) NTRs presumably bind to the same site/overlapping sites on the rCHMP2A protein molecule.

The binding of r-CHMP2A to the radiolabeled 5'(-) NTR transcript was not discernible if it was not photoreactive. This is evident from the experiment shown in Fig. 5C (top panel), in which the DEN-2 virus 5'(-) NTR transcript was prepared either with just UTP (lane "-BrU") or its bromo analog, Br-UTP (lane "+BrU"). Further, we also observed that increasing the amount of the $\mathrm{r}$-mCHMP2A protein, resulted in a dose-dependent increase in the observed intensity of the ribonucleoprotein complex (Fig. 5C, middle panel). Finally, incubation of the complex with Proteinase K or V8 protease, resulted in its elimination, as seen in the bottom panel of Fig. 5C (compare the no protease lane 'NP' with the 'V8' and 'PK' lanes). Bovine serum albumin and an unrelated protein carrying $6 x$ Histidine tag, used as control proteins, did not exhibit any binding to the labeled NTRs (data not shown)

We next carried out a yeast-based confirmatory assay to validate the in vitro binding data described above. To this end, we used yeast three-hybrid system wherein each one of the four DEN-2 NTR transcripts $\left[5^{\prime}(+), 3^{\prime}(+), 5^{\prime}(-)\right.$ and $\left.3^{\prime}(-)\right]$ was separately used as the bait RNA and the r-mCHMP2A protein as the prey. In vivo interaction of the bait with prey in the yeast host was monitored by reconstitution of lac $Z$ expression. The data are presented in Fig. 5D. Consistent with the in vitro observations above, we found that the DEN-2 virus 5'(-) and 3'(-) NTRs (Fig. D, sectors 5 and 6, respectively), but not their complements, $5^{\prime}(+)$ and 3'(+) NTRs (Fig. 5D, sectors 2 and 3, respectively) interacted in vivo with $\mathrm{r}$-mCHMP2A protein. The positive (Fig. 5D, sector 4) and negative (Fig. 5D, sector 1) control experiments run in parallel worked as predicted. Taken together, the in vitro and in vivo binding data strongly supports the notion that indeed the $5^{\prime}(-)$ and $3^{\prime}(-)$ NTRs specifically interact with $\mathrm{r}-$ mCHMP2A.

Mammalian cells contain proteins that specifically bind to 3'(-) NTRs: We sought to determine if endogenously expressed proteins manifest 5 '(-) and 3'() NTR-binding activity akin to rCHMP2A. To this end, BHK cell lysates were incubated separately with radiolabeled 5'(-) and 3'(-) NTRs of all four DEN virus serotypes and analyzed by electrophoretic mobility shift assay. The data in Fig. 6 show that 3'(-) NTRs of all four DEN virus serotypes could form RNA-protein complexes (panel A). Quite interestingly, the 5'(-) NTR also generated multiple, discrete retarded complexes. Again, this was true for all four DEN virus serotypes. We next examined the specificity of these interactions by performing competition experiments. The data for the $5^{\prime}(-)$ and $3^{\prime}(-)$ NTR/protein interactions are presented in panels $\mathrm{C}$ and D, respectively (Fig. 6). RNA/protein complex formation by both these NTRs was unaffected by an excess of unlabeled non-specific competitor (lanes 3 in panels $\mathrm{C}$ and $\mathrm{D}$ ). In striking contrast, the complexes formed by radiolabeled 5' (-) NTR and 3' (-) NTR were competed out by the inclusion of an excess of unlabeled 5' (-) NTR (lane 4, panel C) and 3' (-) NTR (lane 4, panel D), respectively, leading to the conclusion that the observed interactions are specific.

\section{DISCUSSION}

A unique feature common to all positive strand RNA viruses is the need to use the same genomic RNA as a template for both translation as well as replication. While the genomic RNA codes for the RNA-dependent replicase activity, the intricate interplay of events that presumably coordinate translation, replication, vesicular trafficking and budding in the virus life cycle, would need the participation of several additional host factors. An emerging body of evidence shows that a multitude of cellular proteins are indeed utilized by plus strand RNA viruses during their life cycle ${ }^{[6,30]}$. Analogous to DNA-based replication systems, cellular factors have been identified that bind to cis-acting elements of these $\mathrm{NTRs}^{[15,17-20,30]}$.

In this study, we used a cocktail of four radiolabeled DEN-2 virus NTRs [5'(+), 3'(+), 5'(-) and $\left.3^{\prime}(-)\right]$ to screen a murine macrophage cDNA expression library. The rationale for using such a cocktail of NTRs 
was that it would allow us to pick out the maximum number of interacting proteins from which the previously recognized ones could be eliminated and novel ones pursued. Quite interestingly, our search identified for the first time, a $\sim 25 \mathrm{kDa}$ murine macrophage protein belonging to a unique family of mammalian endosome-related proteins known as the class E Vps proteins ${ }^{[28]}$. The primary structure of this protein, predicted from the gene sequence, showed it to be $>99 \%$ identical to human CHarged Multivesicular body Protein, CHMP2A (also known as CHromatin Modifying Protein 2A or BC-2). CHMP2A is a cellular factor, one of a recently recognized family of human proteins involved in sorting proteins into vesicles that bud into multi-vesicular bodies, $\mathrm{MVBs}^{[31,32]}$. The CHMP proteins are localized both in the nucleus and cytoplasm. In the nucleus they are implicated in gene silencing and in the cytoplasm, they function in the MVB pathway ${ }^{[23,33]}$. The MVB pathway is implicated in several key processes, including receptor downregulation, cell growth control, developmental signaling and antigen presentation ${ }^{[28]}$. Recent reports that delineated a network of protein-protein interactions in the human MVB pathway showed that this entire network may participate in the release of enveloped viruses from infected cells ${ }^{[31,32]}$. CHMP2A functions late in the MVB pathway and its deletion/mutation can arrest the release of human immunodeficiency virus 1 from infected cells ${ }^{[31]}$.

To investigate the CHMP2A protein further, we expressed a $6 x-H i s$ tagged version of the protein in $E$. coli and purified it using Ni-NTA affinity chromatography. We then carried out a careful evaluation of the NTR-binding binding properties of this recombinant protein, r-mCHMP2A, using both in vitro and in vivo assays. In vitro binding assays were carried out in two different formats. In one, we used rmCHMP2A immobilized on Ni-NTA beads (liquid assay) and in the other we used a UV-CL approach. The liquid assay showed that the 5' and 3' minus sense NTRs of all four DEN virus serotypes, but not their plus sense complements, bound to Ni-NTA beadimmobilized r-mCHMP2A protein. That this binding occurred in the presence of high concentrations of nonspecific competitors suggests that it is specific. The liquid assay data were reconfirmed using DEN-2 virus 5'(+), 3'(+), 5'(-) and 3'(-) NTRs in the UV-CL assay. Also, based on the successful elimination of the radiolabeled 3' (-) NTR from its r-mCHMP2A complex using a 20-fold molar excess of either unlabeled 5'(-) or 3'(-) NTR RNA suggested that both the minus sense NTRs share the same or overlapping binding sites on the r-mCHMP2A molecule. Finally, using DEN-2 virus NTR-encoding 'bait' and r-mCHMP2A-encoding 'prey' constructs in a yeast three-hybrid assay, we were able to corroborate the in vitro binding data in vivo.

We believe that the DEN virus minus sense $\mathrm{NTR} / \mathrm{mCHMP} 2 \mathrm{~A}$ binding is a specific and significant interaction for the following reasons. First, soluble rmCHMP2A protein manifested binding to the (-) NTRs of all four DEN virus serotypes, in the presence of high concentrations of non-specific inhibitors. This binding was not manifested with any one of the eight (+) NTRs tested. Second, UV-CL experiments showed that radiolabeled DEN-2 virus 5'(-) and 3'(-) NTRs specifically bound to $\mathrm{r}-\mathrm{mCHMP} 2 \mathrm{~A}$. That this binding is specific is evident from the failure of radioactive complex formation in the presence of unlabeled 5'(-) and 3'(-) NTRs. This complex is unaffected by nonhomologous (vector-derived) RNA transcripts. Third, the in vitro binding data are validated by demonstration of in vivo binding of mCHMP2A to DEN-2 virus 5'(-) and 3'(-) NTRs, using a yeast-based assay. Taken together, the in vitro and in vivo observations made so far, suggest that the mCHMP2A protein may preferentially bind only to the ends of the minus sense DEN RNAs. It could be argued that all our binding data has been generated using $E$. coli-expressed recombinant protein and may not reflect the behaviour of endogenous proteins. To address this, we tested the capacity of BHK cell lysates to bind to 5'(-) and 3'(-) NTRs corresponding to all four DEN virus serotypes in EMSAs. Consistent with our prediction, we found that all these minus sense NTRs did indeed generate discrete RNA protein complexes. Further, we could show that these were specific interactions based on competition data (Fig. 6). However, the presence of CHMP2A in these complexes must await the generation of specific, high-affinity antibodies for supershift and coimmunoprecipitation analyses.

Flavivirus replication remains to be elucidated in detail. It was hitherto believed that flavivirus replication occurs exclusively in the cytosol. However, a recent report has shown that a significant proportion of replication activity occurs in the nucleus as well ${ }^{[34]}$. In regard to the mechanism of replication, the current perception envisages an RNA duplex (comprised of the plus and minus sense RNAs), as the putative replicative intermediate, which serves as the template for plus sense genomic RNA synthesis. While host cellular factors capable of binding to the 5' and 3' NTRs of the plus sense flaviviral RNA and to the 3' NTR of the minus sense RNA intermediate have been identified there is no report so far regarding any host protein binding to the 5' NTR of the minus RNA. In this context, our data presented above, implicating 5'(-) NTR also in RNA-protein interaction is intriguing, particularly as there is no experimental evidence to date for the existence of single-stranded minus sense RNA in the flavivirus-infected cell. However, proteins capable of interacting with the 3'(-) NTR of West Nile virus have been found and implicated in the initiation of plus RNA synthesis ${ }^{[22]}$. We could interpret this as indicative of the existence of single stranded minus sense RNA in the flavivirus-infected cell. In this context, our observation of (-) NTR binding activity in 
cell lysates, also points to the existence of minus sense RNA in the DEN virus-infected cells. Presumably the low concentrations and the transient existence of minus sense RNA in single-stranded form have precluded its definitive identification in infected cells.

While the precise significance of the interaction between DEN-2 virus 5' and 3'(-) NTRs and the $\mathrm{mCHMP} 2 \mathrm{~A}$ protein is not clear at the present time, the identification of CHMP2A as a dengue virus minus RNA-binding protein has revealed the possibility of potentially novel role for it and perhaps the MVB pathway, in the life cycle of dengue viruses. This notion draws support from the finding that the MVB pathway protein Tsg101, a class E Vps protein like CHMP2A, co-localizes with the NS3 protein in Japanese encephalitis virus-induced membrane entities ${ }^{[35]}$. The CHMP2A like protein is also recruited by HIV-1 and Ebola virus in infected cells to facilitate virus budding ${ }^{[31,32]}$. It has been suggested that perhaps one of the reasons that the flavivirus replication components are sequestered into membrane protected VPs may be to evade the host mediated response to the dsRNA replicative intermediate through the interferon pathway and RNA interference ${ }^{[36]}$. This is consistent with a recent report, which showed that actively replicating West Nile virus is resistant to cytoplasmic delivery of siRNA $^{[37]}$. Further experiments are needed to decipher the biological relevance of CHMP2A interaction with terminal ends of dengue virus minus RNA in dengue virus life cycle.

\section{ACKNOWLEDGEMENTS}

This work was supported by internal funds from ICGEB and a grant from Department of Biotechnology, Govt. of India. SC is supported by the Council of Scientific and Industrial Research, Govt. of India.

\section{REFERENCES}

1. Gubler, D.J., 1998. Dengue and dengue hemorrhagic fever. Clin. Microbiol. Rev., 11: 480496.

2. Gubler, D.J0, 2002. Epidemic dengue/dengue hemorrhagic fever as a public health, social and economic problem in the 21 st century. Trends Microbiol., 10: 100-103.

3. Lindenbach, B.D. and C.M. Rice, 2001, Flaviviridae: The Viruses and Their Replication. Fields Virology. 4th (Eds D. M. Knipe and P. M. Howley), pp: 991-1041. Lippincot Williams and Wilkins, Philadelphia.

4. Grun, J.B. and M.A. Brinton, 1988. Separation of functional West Nile virus replication complexes from intracellular membrane fragments. J. Gen. Virol., 69 (Pt 12): 3121-3127.
5. Chu, P.W. and E.G. Westaway, 1992. Molecular and ultrastructural analysis of heavy membrane fractions associated with the replication of Kunjin virus RNA. Arch. Virol., 125: 177-191.

6 Ahlquist, P., A.O. Noueiry, W.M. Lee, D.B. Kushner and B.T. Dye, 2003. Host factors in positive-strand RNA virus genome replication. J. Virol., 77: 8181-8186.

7. Mackenzie, J.M., M.K. Jones and P.R. Young, 1996. Immunolocalization of the dengue virus nonstructural glycoprotein NS1 suggests a role in viral RNA replication. Virology, 220: 232-240.

8. Mackenzie, J.M., M.K. Jones and P.R. Young, 1996. Improved membrane preservation of flavivirus-infected cells with cryosectioning. J. Virol., Methods, 56: 67-75.

9. Mackenzie, J.M., A.A. Khromykh, M.K. Jones and E.G. Westaway, 1998. Subcellular localization and some biochemical properties of the flavivirus Kunjin nonstructural proteins NS2A and NS4A. Virology, 245: 203-215.

10. Westaway, E.G., J.M. Mackenzie, M.T. Kenney, M.K. Jones and A.A. Khromykh, 1997. Ultrastructure of Kunjin virus-infected cells: colocalization of NS1 and NS3 with doublestranded RNA and of NS2B with NS3, in virusinduced membrane structures. J. Virol., 71: 66506561.

11. Brinton, M.A. and J.H. Dispoto, 1988. Sequence and secondary structure analysis of the 5'-terminal region of flavivirus genome RNA. Virology, 162: 290-299.

12. Brinton, M.A., A.V. Fernandez and J.H. Dispoto, 1986. The 3'-nucleotides of flavivirus genomic RNA form a conserved secondary structure. Virology, 153: 113-121.

13. Shi, P.Y., M.A. Brinton, J.M. Veal, Y.Y. Zhong and W.D. Wilson, 1996. Evidence for the existence of a pseudoknot structure at the 3 ' terminus of the flavivirus genomic RNA. Biochemistry, 35: 42224230.

14. Shi, P.Y., W. Li and M.A. Brinton, 1996. Cell proteins bind specifically to West Nile virus minusstrand 3' stem-loop RNA. J. Virol., 70: 6278-6287.

15. Ali, N. and A. Siddiqui, 1995. Interaction of polypyrimidine tract-binding protein with the 5' noncoding region of the hepatitis $\mathrm{C}$ virus RNA genome and its functional requirement in internal initiation of translation. J. Virol., 69: 6367-6375.

16. Ali, N. and A. Siddiqui, 1997. The La antigen binds 5 ' noncoding region of the hepatitis $\mathrm{C}$ virus RNA in the context of the initiator AUG codon and stimulates internal ribosome entry site-mediated translation. Proc. Natl. Acad. Sci. USA, 94: 22492254. 
17. Chen, C.J., M.D. Kuo, L.J. Chien, S.L. Hsu, Y.M. Wang and J.H. Lin, 1997. RNA-protein interactions: involvement of NS3, NS5 and 3' noncoding regions of Japanese encephalitis virus genomic RNA. J. Virol., 71: 3466-3473.

18. Ito, T. and M.M. Lai, 1997. Determination of the secondary structure of and cellular protein binding to the 3'-untranslated region of the hepatitis $\mathrm{C}$ virus RNA genome. J. Virol., 71: 8698-8706.

19. Tsuchihara, K., T. Tanaka, M. Hijikata, S. Kuge, H. Toyoda, A. Nomoto, N. Yamamoto and K. Shimotohno, 1997. Specific interaction of polypyrimidine tract-binding protein with the extreme 3'-terminal structure of the hepatitis $\mathrm{C}$ virus genome, the 3 'X. J. Virol., 71: 6720-6726.

20. Blackwell, J.L. and M.A. Brinton, 1997. Translation elongation factor- 1 alpha interacts with the 3' stem-loop region of West Nile virus genomic RNA. J. Virol., 71: 6433-6444.

21. Ta, M. and S. Vrati, 2000. Mov34 protein from mouse brain interacts with the 3 ' noncoding region of Japanese encephalitis virus. J. Virol., 74: 51085115.

22. Li, W., Y. Li, N. Kedersha, P. Anderson, M. Emara, K.M. Swiderek, G.T. Moreno and M.A. Brinton, 2002. Cell proteins TIA-1 and TIAR interact with the $3^{\prime}$ stem-loop of the West Nile virus complementary minus-strand RNA and facilitate virus replication. J. Virol., 76: 1198912000.

23. Howard, T.L., D.R. Stauffer, C.R. Degnin and S.M. Hollenberg, 2001. CHMP1 functions as a member of a newly defined family of vesicle trafficking proteins. J. Cell Sci., 114: 2395-2404.

24. Fujita, H., Y. Umezuki, K. Imamura, D. Ishikawa, S. Uchimura, A. Nara, T. Yoshimori, Y. Hayashizaki, J. Kawai, K. Ishidoh, Y. Tanaka and M. Himeno, 2004. Mammalian class E Vps proteins, SBP1 and $\mathrm{mVps} 2 / \mathrm{CHMP} 2 \mathrm{~A}$, interact with and regulate the function of an AAA-ATPase SKD1/Vps4B. J. Cell Sci., 117: 2997-3009.

25. Sagesser, R., E. Martinez, M. Tsagris and M. Tabler, 1997. Detection and isolation of RNAbinding proteins by RNA-ligand screening of a cDNA expression library. Nucleic Acids Res., 25: 3816-3822.

26. AnandaRao, R., S. Swaminathan, S. Fernando, A.M. Jana and N. Khanna, 2005. A customdesigned recombinant multiepitope protein as a dengue diagnostic reagent. Protein Expr. Purif., 41: 136-147.
27. Keesee, S.K., R. Obar and Y.J. Wu, 1999. Materials and methods for detection of breast cancer. US Patent, 5: 914, 238.

28. Katzmann, D.J., G. Odorizzi and S.D. Emr, 2002. Receptor downregulation and multivesicular-body sorting. Nat. Rev. Mol. Cell Biol., 3: 893-905.

29. Golovanov, A.P., G.M. Hautbergue, S.A. Wilson and L.Y. Lian, 2004. A simple method for improving protein solubility and long-term stability. J. Am. Chem. Soc., 126: 8933-8939.

30. Lai, M.M., 1998. Cellular factors in the transcription and replication of viral RNA genomes: a parallel to DNA-dependent RNA transcription. Virology, 244: 1-12.

31. von Schwedler, U.K., M. Stuchell, B. Muller, D.M. Ward, H.Y. Chung, E. Morita, H.E. Wang, T. Davis, G.PJ. He, D.M. Cimbora, A. Scott, H.G. Krausslich, J. Kaplan, S.G. Morham and W.I. Sundquist, 2003. The protein network of HIV budding. Cell, 114: 701-713.

32. Martin-Serrano, J., A. Yarovoy, D. Perez-Caballero and P.D. Bieniasz, 2003. Divergent retroviral latebudding domains recruit vacuolar protein sorting factors by using alternative adaptor proteins. Proc. Natl. Acad. Sci. USA, 100: 12414-12419.

33. Stauffer, D.R., T.L. Howard, T. Nyun and S.M. Hollenberg, 2001. CHMP1 is a novel nuclear matrix protein affecting chromatin structure and cell-cycle progression. J. Cell. Sci., 114: 23832393.

34. Uchil, P.D., A.V. Kumar and V. Satchidanandam, 2006. Nuclear localization of flavivirus RNA synthesis in infected cells. J. Virol., 80: 5451-5464.

35. Chiou, C.T., C.C. Hu, P.H. Chen, C.L. Liao, Y.L. Lin and J.J. Wang, 2003. Association of Japanese encephalitis virus NS3 protein with microtubules and tumour susceptibility gene 101 (TSG101) protein. J. Gen. Virol., 84: 2795-2805.

36. Uchil, P.D. and V. Satchidanandam, 2003. Architecture of the flaviviral replication complex. Protease, nuclease and detergents reveal encasement within double-layered membrane compartments. J. Biol. Chem., 278: 24388-24398.

37. Geiss, B.J., T.C. Pierson and M.S. Diamond, 2005. Actively replicating West Nile virus is resistant to cytoplasmic delivery of siRNA. Virol. J., 2: 53. 